Case

Report

\title{
Late Stent Graft Infection after the Emergency Endovascular Repair of a Secondary Iliac Artery-Enteric Fistula Treated with Graft Removal and In Situ Aortic Reconstruction Using Femoral Veins
}

\author{
Yohei Yamamoto, MD, Kimihiro Igari, MD, PhD, Takahiro Toyofuku, MD, PhD, \\ Toshifumi Kudo, MD, PhD, and Yoshinori Inoue, MD, PhD
}

\begin{abstract}
An arterioenteric fistula is a devastating and life-threatening condition that requires urgent treatment. Less-invasive endovascular treatment has emerged as an alternative to conventional open repair, but postoperative graft infection remains a major concern. We herein report a case of late stent graft infection after emergency endovascular repair of a secondary iliac artery-enteric fistula. The patient was a 63-year-old male who presented with a fever, who had undergone successful endovascular stent grafting for a secondary common iliac artery-enteric fistula 29 months prior. The diagnosis of a stent graft infection was confirmed via computed tomography. He underwent graft removal and in situ reconstruction with femoral vein grafts. At 6-month follow-up, the patient is in a good general condition without any symptoms.
\end{abstract}

Keywords: arterioenteric fistula, endovascular repair, stent graft, infection, femoral vein

\section{Introduction}

Endovascular treatment for arterioenteric fistulas (AEFs) has emerged as a less-invasive treatment modality. Endovascular treatment has lower perioperative morbidity and mortality than open repair, but the high incidence of recurrent bleeding and infection make it difficult to recommend across the board. We herein report a case of late stent graft infection after emergency endovascular repair of a secondary common iliac artery-enteric fistula. Infected graft removal and in situ aortic reconstruction with femoral

Division of Vascular and Endovascular Surgery, Department of Surgery, Tokyo Medical and Dental University, Tokyo, Japan

Received: May 26, 2016; Accepted: June 15, 2016

Corresponding author: Yohei Yamamoto, MD. Division of Vascular Surgery, Department of Surgery, Tokyo Medical and Dental University, 1-5-45 Yushima, Bunkyo-ku, Tokyo 113-8519, Japan Email: y-yamamoto.srg1@tmd.ac.jp

(C)2017 The Editorial Committee of Annals of Thoracic and Cardiovascular Surgery. All rights reserved. vein $(\mathrm{FV})$ grafts were performed in our case. We also discuss the role of endovascular treatment for AEFs and the management of aortic graft infection according to the published literature.

\section{Case Report}

A 63-year-old male presented with a chief complaint of a fever. He had a history of multiple surgical revascularization due to the right persistent sciatic artery causing lower extremity ischemia at another hospital. The last operation in a series was aneurysm resection and polytetrafluoroethylene (PTFE) patch angioplasty for pseudoaneurysm at the proximal anastomosis site of the previous right common ilio-popliteal artery bypass 10 years before the current presentation. Twenty-nine months prior, he had presented to the emergency room with repeated hematochezia. He was subjected to urgent computed tomography (CT), which revealed the presence of a pseudoaneurysm at the right common iliac artery in close apposition to a segment of 


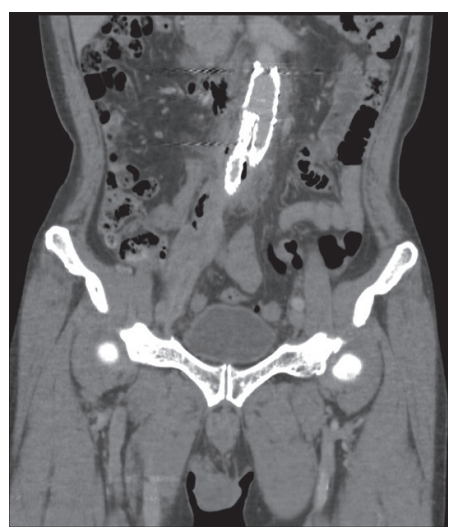

B

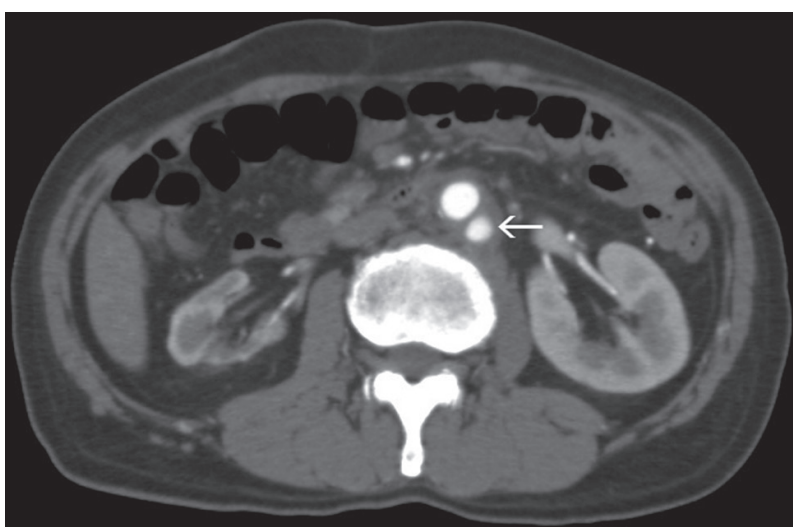

Fig. 1 (A) A CT image showing gas bubbles around the stent graft and periaortic soft tissue attenuation. (B) A CT image 16 days after starting the conservative treatment for stent graft infection showing the development of an aortic pseudoaneurysm (arrow).

small intestine. We decided to proceed with endovascular repair, although the findings on arrival were consistent with an AEF, because severe intraabdominal adhesion formation following multiple surgical intervention was suspected. We ran into an anatomic access problem, as the patient did not have the right femoral artery associated with the persistent sciatic artery. We therefore planned to use access via the left femoral and left axillary arteries to perform the procedure.

The operation was performed under general anesthesia. The main body of the stent graft (Gore Excluder PXT231212; W.L. Gore \&. Associates Inc., Flagstaff, AZ, USA) was introduced via left femoral access and deployed above the aortic bifurcation. A 16-Fr 68.6-cm sheath was advanced into the right internal iliac artery via left axillary access. Given the small diameter of the right internal iliac artery $(9 \mathrm{~mm})$, the upside-down Excluder contralateral leg (PXC121400), which was extracorporeally predeployed in the 12-Fr sheath, was positioned and deployed.

After the procedure, the patient's hematochezia soon disappeared. Intravenous antibiotic treatment was continued for two weeks, followed by a further course of oral antibiotics for three months. He was discharged on postoperative day 41 . His postoperative course was uneventful, with no local or systemic signs of infection over the next two years, until he presented with a fever.

On arrival at our facility, his temperature was $37.9^{\circ} \mathrm{C}$, but his other vital signs were otherwise unremarkable. The findings from laboratory examinations showed a white blood cell (WBC) count of 11200 cells $/ \mathrm{mm}^{3}$ and a C-reactive protein (CRP) level of $17.75 \mathrm{mg} / \mathrm{dL}$. Enhanced CT showed gas bubbles around the stent graft and periaortic soft tissue attenuation that suggested a graft infection (Fig. 1A).
A conservative treatment was initiated with intravenous meropenem and vancomycin. He became afebrile within a few days, and two weeks after the initiation of the antibiotic treatment, his WBC count dropped to normal, and his CRP level decreased to $2.89 \mathrm{mg} / \mathrm{dL}$. The blood cultures obtained during the conservative treatment were negative. On Day 16 after starting treatment, a follow-up CT scan was performed, which revealed an aortic pseudoaneurysm around the proximal portion of the stent graft that had not been present on previous studies (Fig. 1B). A decision was made to surgically remove the stent graft due to the risk of rupture and persistent infection.

Under general anesthesia, long midline laparotomy was performed. After peeling off the intraperitoneal adhesion, the infrarenal aorta, right internal iliac artery, left internal iliac artery, and external iliac artery were controlled. A segment of the small intestine was strongly adherent to the right common iliac artery, and an AEF was found between them (Fig. 2). Segmental resection of the involved small intestine with primary anastomosis was performed. Both FVs were harvested from the popliteal fossa to the origin of the FV with preservation of the deep FV for venous drainage. The infected stent graft was then removed, and end-to-end anastomosis between the aorta and the reversed FV was performed. The graft was brought down, and distal end-to-end anastomosis with the right internal iliac artery was performed. The other FV was anastomosed proximally onto the graft in a side-to-end fashion and distally onto the left external iliac artery in an end-to-side fashion. The cultures of the tissue samples collected from the infected fields were negative. 

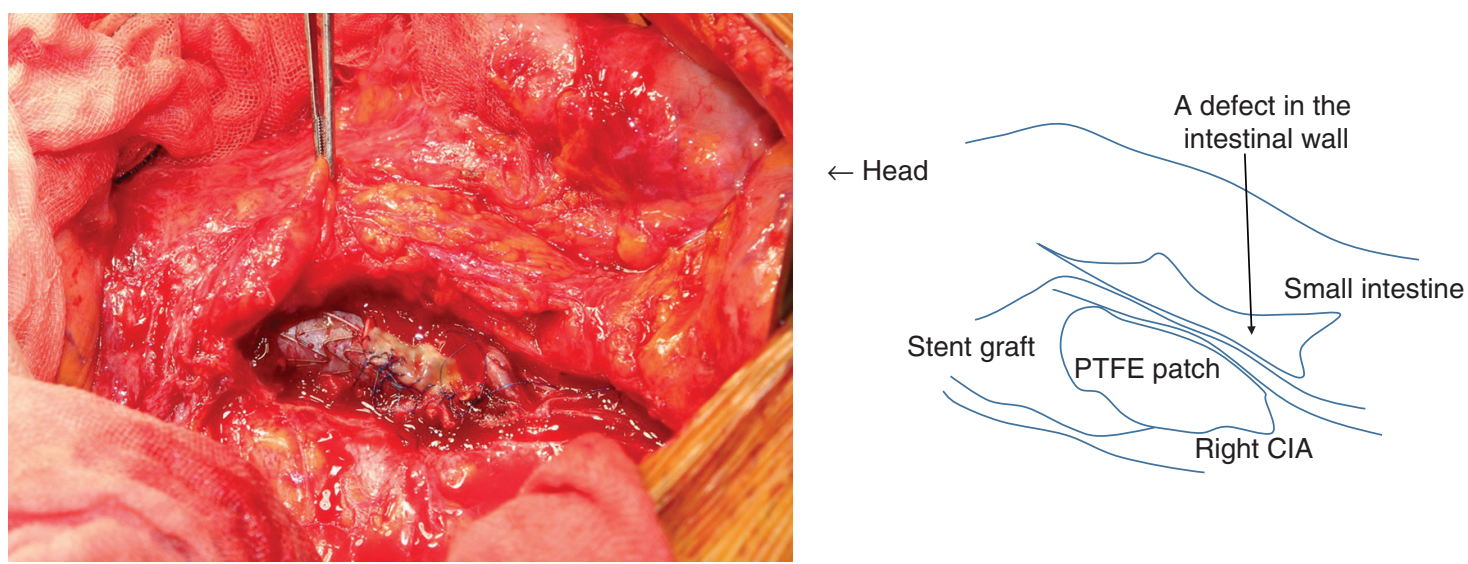

Fig. 2 An intraoperative image showing a defect in the intestinal wall and the disruption of the suture line of the PTFE patch with exposure of the stent graft. PTFE: polytetrafluoroethylene; CIA: common iliac artery

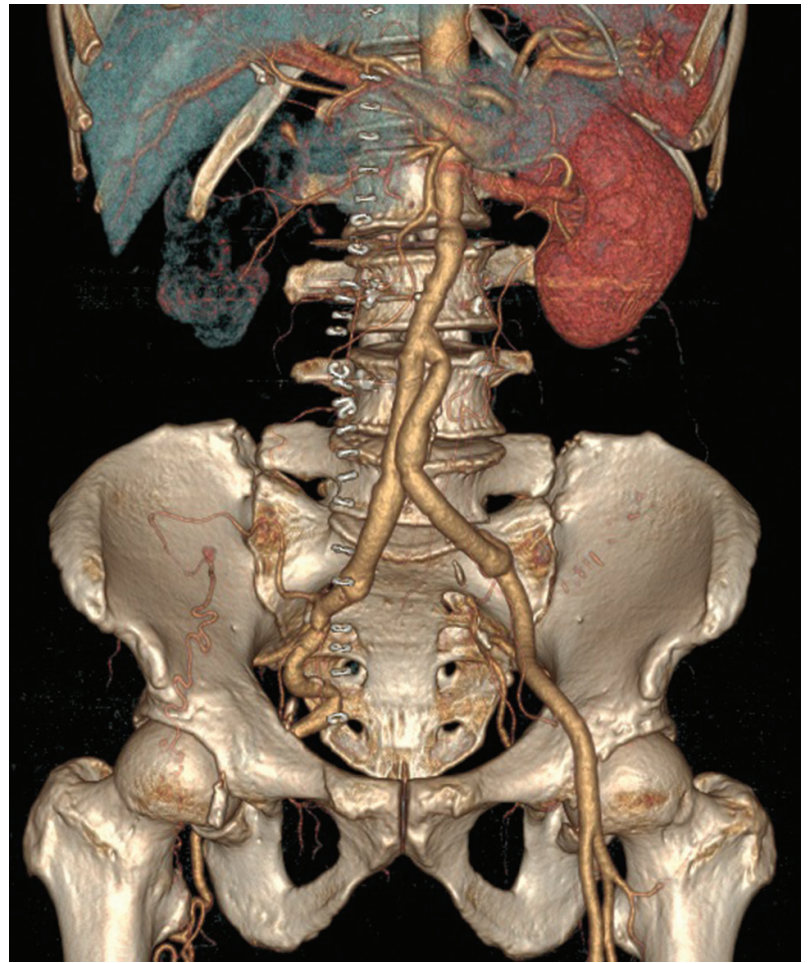

Fig. 3 A postoperative CT image showing successful aortic reconstruction.

The postoperative period was complicated by mild paralytic ileus which settled with conservative treatment. No venous morbidities associated with the FV harvest were observed. Intravenous meropenem and vancomycin were continued for 34 days after surgery, followed by a further 11 days of oral levofloxacin. A postoperative CT scan showed successful aortic reconstruction (Fig. 3). The patient was discharged 47 days after surgery. At 6 months' follow up, he resumed a normal life with no complaints.

\section{Discussion}

The highlights of our case are the endovascular treatment of an AEF and management of an aortic graft infection.

AEFs are a devastating condition with a high probability of mortality. Primary AEFs are caused by aneurysms, infection, malignancy, or ingested foreign objects and are rarer than secondary AEFs. ${ }^{1)}$ Most AEFs involve the proximal anastomotic site at the abdominal aorta and the third or fourth portions of the duodenum, with an incidence of around or even less than $1 \%$ after aortic surgery. ${ }^{2}$ Iliac artery-enteric fistulas (IEFs) are rare types of AEFs.

In a review of IEF-focused manuscripts published up to 1987, it was reported that $55 \%$ of the IEFs were due to atherosclerotic aorto-iliac aneurysms. ${ }^{3)}$ By contrast, in a review of IEF-focused manuscripts published from 1990 to $2015,{ }^{4)} 41 \%$ of the IEFs were associated with prior nonvascular abdominal or pelvic surgical intervention. Other predisposing factors included prior aortic intervention, pelvic malignancy, and intraabdominal or vascular infection. The most frequently involved artery is the common iliac artery (44\%), and the most frequently involved bowel segment is the colon (51\%). Almost all the patients with IEFs present with lower gastrointestinal bleeding. The reported 30-day mortality rate is $26 \%$, including $20 \%$ of all deaths occurring intraoperatively.

Conventional open surgical repair of AEFs in the acute setting is associated with significant morbidity and mortality. In the last two decades, less-invasive endovascular procedures have emerged as an alternative treatment option for disease in the aorta and iliac arteries. Several reports have described the endovascular repair of AEFs. Antoniou et al. ${ }^{5)}$ observed a graft infection or recurrent bleeding in $44 \%$ of 
patients after endovascular repair of AEFs and a mortality rate of $29 \%$ during a mean follow-up of 13 months. In a report by Kakkos et al., ${ }^{6}$ ) no in-hospital deaths were observed after endovascular repair of AEFs, but the in-hospital mortality rate was $35 \%$ after open repair. Despite the short-term benefits, sepsis and recurrent bleeding generally worsened the 2-year overall survival rate in the endovascular repair group (24\%) compared with the open repair group (50\%). Some authors have reported the utility of endovascular repair as a bridge procedure to definitive surgical repair., ${ }^{7,8)}$ Consequently, most authors refer to endovascular repair as a possible treatment option for patients who are not candidates for open surgery or a bridge to definitive open repair under more optimized conditions. ${ }^{9)}$

In the present case, a common iliac artery-small intestine fistula and a pseudoaneurysm were successfully controlled with endovascular stent grafting, and no complications were observed in the perioperative period. However, a graft infection occurred 29 months after the graft implantation. Our experience, coupled with previous reports on endovascular repair of AEFs, suggests that definitive open repair under stable hemodynamics should be considered after emergency endovascular repair of AEFs, even if it appears that the conditions have been completely controlled, in patients who have two or more years of life expectancy without significant comorbidities.

Another clinical challenge that we faced in our case was management of the aortic graft infection. With a growing number of patients being treated with stent grafts, the management of stent graft infections has recently been discussed in a similar way to graft infections following conventional open aneurysm repair. ${ }^{10)}$ In addition to adequate antibiotic therapy, the management of aortic graft infections consists of the removal of the infected graft and revascularization. Traditionally, revascularization has been accomplished via extra-anatomic bypass, usually axillobifemoral bypass, but due to the significant morbidity and mortality of this approach, in situ reconstruction with rifampicin-bonded grafts, cryopreserved allografts, or autologous FV grafts have been developed. ${ }^{11)}$ In the present case, the patient was successfully treated by removing the infected graft and performing in situ reconstruction with FV grafts. Aortic reconstruction with FV grafts was first introduced in 1993 by Clagett et al. ${ }^{12}$ and Nevelsteen et al. ${ }^{13)}$ The reported 30-day mortality rate is less than $10 \%$, with a 5-year survival rate of 50\%-60\% and a 5-year limb salvage rate of about $90 \% .{ }^{14,15)}$ The reinfection rate is below $2 \%,{ }^{16)}$ indicating that reconstruction with the FV grafts is the most effective method of preventing reinfection. The mid- to long-term venous morbidity after an FV harvest is minimal. ${ }^{17)}$ The major drawback to this technique is the long operation time. ${ }^{11)}$ In the present case, we prioritized a resistance to infection when choosing the graft material, as the causative organism was not identified pre- or intraoperatively. In addition, the greater omentum, which usually plays an important role in preventing graft infections, had already been used for the previous operation.

\section{Conclusion}

Graft infection remains a possible long-term complication after endovascular treatment for an AEF. Definitive open repair should be considered in young patients with good life expectancy and no significant comorbidities. The removal of the infected graft and in situ reconstruction with FV grafts is a valuable surgical treatment.

\section{Disclosure Statement}

All authors have no conflict of interest.

\section{References}

1) Saers SJ, Scheltinga MR. Primary aortoenteric fistula. Br J Surg 2005; 92: 143-52.

2) Bergqvist D, Björck M. Secondary arterioenteric fistulation-a systematic literature analysis. Eur J Vasc Endovasc Surg 2009; 37: 31-42.

3) Vetto JT, Culp SC, Smythe TB, et al. Iliac arterialenteric fistulas occurring after pelvic irradiation. Surgery 1987; 101: 643-7.

4) Policha A, Baldwin M, Mussa F, et al. Iliac artery-uretero-colonic fistula presenting as severe gastrointestinal hemorrhage and hematuria: a case report and review of the literature. Ann Vasc Surg 2015; 29: 1656: e1-6.

5) Antoniou GA, Koutsias S, Antoniou SA, et al. Outcome after endovascular stent graft repair of aortoenteric fistula: A systematic review. J Vasc Surg 2009; 49: 782-9.

6) Kakkos SK, Antoniadis PN, Klonaris CN, et al. Open or endovascular repair of aortoenteric fistulas? A multicentre comparative study. Eur J Vasc Endovasc Surg 2011; 41: 625-34.

7) Franchin M, Tozzi M, Piffaretti G, et al. Emergency endovascular "bridge" treatment for iliac-enteric fistula. Cardiovasc Intervent Radiol 2011; 34: 1106-8.

8) Marone EM, Mascia D, Kahlberg A, et al. Emergent endovascular treatment of a bleeding recurrent aortoenteric fistula as a "bridge" to definitive surgical repair. J Vasc Surg 2012; 55: 1160-3.

9) Setacci C, de Donato G, Setacci F. Endografts for the treatment of aortic infection. Semin Vasc Surg 2011; 24: 242-9. 
10) Setacci C, Chisci E, Setacci F, et al. How to diagnose and manage infected endografts after endovascular aneurysm repair. Aorta (Stamford) 2014; 2: 255-64.

11) Berger P, Moll FL. Aortic graft infections: is there still a role for axillobifemoral reconstruction? Semin Vasc Surg 2011; 24: 205-10.

12) Clagett GP, Bowers BL, Lopez-Viego MA, et al. Creation of a neo-aortoiliac system from lower extremity deep and superficial veins. Ann Surg 1993; 218: 239-48; discussion 248-9.

13) Nevelsteen A, Lacroix H, Suy R. The superficial femoral vein as autogenous conduit in the treatment of prosthetic arterial infection. Ann Vasc Surg 1993; 7: 556-60.
14) Ali AT, Modrall JG, Hocking J, et al. Long-term results of the treatment of aortic graft infection by in situ replacement with femoral popliteal vein grafts. J Vasc Surg 2009; 50: 30-9.

15) Heinola I, Kantonen I, Jaroma M, et al. Editor's choice - treatment of aortic prosthesis infections by graft removal and in situ replacement with autologous femoral veins and fascial strengthening. Eur J Vasc Endovasc Surg 2016; 51: 232-9.

16) Chung J, Clagett GP. Neoaortoiliac System (NAIS) procedure for the treatment of the infected aortic graft. Semin Vasc Surg 2011; 24: 220-6.

17) Wells JK, Hagino RT, Bargmann KM, et al. Venous morbidity after superficial femoral-popliteal vein harvest. J Vasc Surg 1999; 29: 282-9; discussion 289-91. 